\title{
RESEARCH
}

Open Access

\section{Prison sentencing increases the risk of unemployment among illegal heroin users in Taiwan}

Charles Tzu-Chi Lee ${ }^{1 \dagger}$, Chiu-Mieh Huang ${ }^{2 \dagger}$, Li-Chun Chang ${ }^{3}$, Shih-Wen Wang ${ }^{1}$, Hsiao-Pei Hsu ${ }^{2}$, Jung-Yu Liao ${ }^{4}$ and Jong-Long Guo ${ }^{1 *}$ (D)

\begin{abstract}
Background: Previous studies have rarely explored the effect of type of sentencing on employment status among illegal heroin users, therefore, we aims to examine the association of the sentencing types and employment outcomes among illegal heroin users in Taiwan.

Methods: Participants with illegal heroin use were identified through the national prison register system and deferred prosecution system: 2406 with deferred prosecutions, 4741 with observation and rehabilitation, 15 compulsory rehabilitation and 1958 sentenced to prison in calendar 2011. Logistic regression models were built to estimate the effect of sentencing type on unemployment status at 2 years after release. Stratification analysis was conducted to determine the effect of sentencing type based on the offender's employment status before sentencing.
\end{abstract}

Results: Illegal heroin users receiving a prison sentence were more than twice as likely to be unemployed 2 years later than those receiving deferred prosecution. The unemployment rate was also higher for those with observation and rehabilitation and compulsory rehabilitation than deferred prosecution in the 2 years following sentencing. Males, older users, without a job before sentencing, divorced or widowed and higher prior drug use criminal records were also higher risk of unemployment. Subgroup analysis by prior employment status revealed that being sentenced to prison, observation and rehabilitation and compulsory rehabilitation affected the subsequent employment status only for those heroin users with a job before sentencing. The strength of associations showed dose-dependent relationship between different sentencing types (sentenced to prison> compulsory rehabilitation> observation and rehabilitation) and employment outcomes.

Conclusions: Illegal heroin users who receive a prison sentence have a much higher risk of unemployment than those who receive deferred prosecution after controlling potential confounders, especially those who had a job before sentencing. The implication is the stronger freedom of punishment, the higher risk of unemployment outcomes. Our study support that illegal heroin user is legally regarded as a patient before being regarded as a criminal, so giving priority to quit addition rather than imprisonment.

Keywords: Deferred prosecution, Observation and rehabilitation, compulsory rehabilitation, Prison sentence, Employment rate, Heroin users

\footnotetext{
* Correspondence: jonglong@ntnu.edu.tw

${ }^{+}$Charles Tzu-Chi Lee and Chiu-Mieh Huang contributed equally to this work. 'Department of Health Promotion and Health Education, National Taiwan Normal University, 162, Sec. 1, He-ping East Road, Taipei, Taiwan 10610 Full list of author information is available at the end of the article
} 


\section{Background}

Illegal drug use and addiction represent a global crisis, creating seriously harm to the addict's physical and mental health [1], social function and employment [2], even endangering the social order by increasing the rates of both crime and violence [3, 4]. For these reasons, numerous countries are committed to implementing programs to prevent, control and treat the use of illegal drugs. In 2018, according to the 2020 World Drug Report, 269 million people aged 15-64 years used at least one illegal drug in the previous year, an estimated 5.4\% of the population; the most used illegal drugs are cannabis, opioids and opiates [5]. The prevalence of illegal drug use in Taiwan was approximate $1.2 \%$, according to Taiwan's first national household survey; the top five most used illegal drugs were methamphetamine, ecstasy, ketamine marijuana and heroin [6]. Heroin in particular is one of the world's most widely used illegal drug [7]. According to the Taiwan Surveillance System of Drug use and Addiction Treatment, heroin is the one of the most commonly used drug in Taiwan; among drugs monitored, heroin accounted for $80.9 \%$ of illegal drug users who received treatment in 2002, a rate which rose to $93.8 \%$ in 2007 , then declined to $83.3 \%$ in 2011 [8]. These percentages were more than $80 \%$ of illegal drug user received treatment.

Use of heroin and other substances burdens both the individual user and society as a whole, to a greater extent than other mental disorders [9]. Physical and mental disorders resulting from illegal heroin use may increase the medical cost, but this represents only be a small portion of the total cost of heroin dependency. For example, one study found that the medical cost of heroin dependency ( $23 \%$ of the total cost) is much smaller than the productivity loss (53\% of the total cost) [10]. To this should be added the cost to the individual users (including financial expenses and health risks) as well as the social costs of crime to support the drug behavior [11].

Previous studies have shown that illegal drug use treatment reduced substance use and criminal behavior and improved the social functioning, employment, and physical and emotional health of the former user [12, 13]. The drug treatment alternative to prison program in Taiwan started as a deferred sentencing model, assisting illegal drug user into treatment. A 5-year study of the program indicated that the re-arrest rates and reconviction rates of those with deferred sentencing were significantly lower than those of offenders who received regular processing into the criminal justice system [14]. Taiwan also initiated a deferred prosecution and methadone maintenance treatment (MMT) pilot project to address the human immunodeficiency virus (HIV) epidemic. A shift in the official view of illegal drug users from "criminal" to "patient" in Taiwan suggested the corresponded strategic approach from "punishment" to "treatment." Before 1998, all illegal drug users in Taiwan were sentenced as "criminals" to criminal penalties. After 1998, the Against Narcotics Act classified illegal drug users as "patients," eligible for treatment and other nonsentencing interventions. Consequently, there was a deferred prosecution policy which did not immediately imprison the illegal drug users, but prefer to provide treatment [15]. Thus, illegal drug users in Taiwan may receive one of four types of sentence: (1) deferred prosecution and complete MMT, (2) observation and rehabilitation, (3) compulsory rehabilitation and (4) prison sentence. This sentencing aim to help illegal heroin users return to society, resume employment and live a normal life. It has replaced imprisonment with medical treatment, offers heroin offenders MMT, improves social interactions, and helps former users transition back into society.

For the illegal heroin user who was first seized by the police, the illegal drug user indicated to the prosecutor that he / she had the intention to quit addiction. If the prosecutor thought that he / she was suitable to stay in the community to quit addiction he / she was commanded to receive deferred prosecution and complete MMT. If the illegal drug user does not express his / her intention to quit addiction, the prosecutor will command the illegal heroin user to receive observation and rehabilitation with a period of less than 2 months. If there is no tendency to indicate the user continue using heroin, he / she will not be prosecuted at the end of the observation and rehabilitation. If there is a tendency to continue to use heroin after professional evaluation, he / she will receive a compulsory rehabilitation which is a treatment with a period more than 6 months until there is no need to continue compulsory rehabilitation. However, it may not exceed 1 year. Recidivists within 5 years may be prosecuted or applied for summary judgment, but prosecutors can still give deferred prosecutions and complete MMT if illegal heroin user indicates to the prosecutor that he or she has an intention to quit addition. Illegal heroin user who receives deferred prosecutions and complete MMT, may be withdrew and prosecuted directly by the prosecutor if any criminal case has been committed during the subsequent two-year period of probation [15].

Currently, Taiwan's prisons have a serious overpopulation problem. In 2014, the estimated available space for each prisoner was only $15.3 \mathrm{ft}^{2}$, far below the $24.9 \mathrm{ft}^{2}$ specified by international human rights regulations [16]. Considering the addictive nature of drugs, not immediately prosecuting drug users, but first giving them an opportunity for treatment, perhaps reduce prison over-crowding and increase the space available to prisoners. 
Employment status can be a critical indicator of social control, economic conditions, return to society and recidivism. Employment is an essential component of healthy social development. Critical to identity formation [17], the accumulation of social capital [18] and perceived quality of life [19], employment can enhance physical and mental well-being and overall quality of life $[20,21]$. Illegal drug use is associated with poor employment outcomes. The unemployment problem of heroin users could be caused by both of the drug-abusing itself (i.e. adverse psychosocial outcomes, including disability or impairment and psychiatric comorbidity) and the social discrimination $[22,23]$. For example, previous study found that the use of heroin and cocaine, as well as the higher frequency of use, is predictive of membership in a low-employment trajectory group through middle adulthood [24]. Evidence-based determinants of employment include educational attainment [25], race [26], marital status and family structure [27], socio-economic status [28] and general health status [29]. However, these studies did not explore whether the type of sentence affected subsequent employment status among illegal heroin users. Therefore, this study examines the association of sentencing types and employment status at 2 years after release among illegal heroin users in Taiwan, to determine the long-term effectiveness of alternatives to prison sentencing.

\section{Method}

\section{Ethics statement}

The Institutional Review Board (IRB) of National Taiwan Normal University approved this study (No. 201606HM002). Written consent from the study participants was waived because the data were collected from a population-based database of de-identified secondary data.

\section{Sample}

This was a population-based cohort study. Illegal heroin users in the national prison register system and deferred prosecution system were identified who received a sentence from January 1, 2011 to December 31, 2011. There were 2406 deferred prosecutions with MMT requirement, 4741 observation and rehabilitation, 15 compulsory rehabilitation and 1958 sentences to prison included in this study. All study participants were followed until 2 years after the index date, that is, the date of deferred prosecution, release from prison or release from the treatment centers.

\section{National Health Insurance in Taiwan}

In 1995, the National Health Insurance (NHI) program, a government-run, single-payer insurance system, was established in Taiwan. By December 2010, 23.074 million people were enrolled nationwide, with a coverage rate of 99.6\%. The NHI Research Database (NHIRD) contains patient demographic and insurance premium data. The insurance premium was used as a proxy indicator of employment status and was classified as with or without job salary. Those with a fixed premium who were also listed as a dependent on the insurance premium were considered as not having a job salary. The fixed premium group is composed of people receiving social welfare support and includes low-income people and veterans. The dependent insurance premium group is comprised of people with family members with no job or income.

\section{Main outcome and study variables}

Two periods were used to measure the employment status of study participants, the follow-up period and the past period. The follow-up period for monitoring employment status began at the date of deferred prosecution or release from either prison or the treatment centers and continued until 2 years after the index date. The past period for monitoring employment status was retrospective, tracking back 2 years before the index date. The past period of employment status was included in the analysis as a control variable. In our database, the minimal follow-up duration after index date for all study subjects is around 2 years. Therefore, we used 2 years as time window to analyze the employ status after index date.

For controlling potential confounders, social demography was collected from National Population Register Database, including gender, age, types of residence (rural, urban), insurance premium (with/without job salary), education level (elementary, junior high school, senior high school and college) and marital status (single, married, divorced and widowed) [22, 30, 31]. Illicit drug use criminal record collected from National Police Agency in Taiwan [32, 33]. Mental diseases including schizophrenia, depressive disorders, bipolar disorders and general health condition (Charlson comorbidity score) were collected from NHIRD [31, 34, 35]. All of the potential comorbid diseases and Charlson comorbidity score were defined using outpatient and inpatient diagnosis before the index date.

\section{Statistical analysis}

Chi-square analysis was used to examine the differences in demographic and other characteristics of those with or without a job at 2 years after the index date. The impact of the type of sentence was analyzed using logistic regression. The un-adjusted and adjusted odds-ratio (AOR) were reported. The model was tested first using all participants, then according to employment status before sentencing. Dummy variables were created to perform collinearity diagnosis by using multiple regression, variance inflation factor (VIF) and condition index were 
reported. Variables with VIF $>10$ or condition index $>30$ was considered as collinearity problem in the study model. Analyses were carried out using SAS version 9.4 (SAS Institute Inc., Cary, NC, USA). $P$ values $<0.05$ were considered statistically significant.

\section{Results}

\section{Sample characteristics}

Results for the characteristics of illegal heroin users are summarized in Table 1. Of the 1848 participants unemployed at 2 years after the index date, 1524 were male and 324 were female. The unemployment rate was similar between males and females $(P=0.759)$ and those with rural and urban residence $(P=0.087)$, schizophrenia $(P=0.321)$, depression $(P=0.687)$, bipolar $(P=0.381)$. The unemployment rate was associated with age, prior employment status, type of sentencing, education level, marital status, illegal drug use criminal record and Charlson comorbidity score (all $P<0.05$ ).

\section{Risk factors associated with unemployment status among illegal heroin users}

After controlling for gender, age, type of residence, previous employment status, education level, marital status, illegal drug use criminal record, schizophrenia, depression, bipolar and Charlson comorbidity score, the unemployment rate at 2 years of those sentenced to prison was 2.30 times that of those who received deferred prosecution $(\mathrm{AOR}=2.30,95 \%$ Confidence Interval $[\mathrm{CI}]$ : 1.97-2.65, $P<0.001)$. The unemployment rate was also higher for those with observation and rehabilitation and compulsory rehabilitation than deferred prosecution in the 2 years following sentencing. Males, older users, without a job before sentencing, divorced or widowed and higher prior illegal drug use criminal records were also higher risk of unemployment. With senior high school educational level was lower significant risk of unemployment. The strength of associations showed dosedependent relationship between different sentencing types (sentenced to prison> compulsory rehabilitation> observation and rehabilitation) and employment outcomes (all $P<0.001$, Table 2). In this study, the dysfunction of mental (schizophrenia, depression and bipolar) and general health condition (Charlson comorbidity score) were not significantly associated with unemployment rate in illegal heroin users (all $p>0.05$, Table 2).

\section{Stratified analysis by prior unemployment status}

There was a significant interaction between prior employment status and type of sentence $(P<0.001)$. To determine the relationship between type of sentence and the prior employment status of participants, we performed stratification analysis. For participants without a job before the index date, the unemployment rate at 2 years after the index date was not significant difference in type of sentencing (Table 3 ). However, at 2 years after sentencing, for those with a job before the index date, the unemployment rate for those who received a prison sentence was higher than for those who received deferred prosecution $(\mathrm{AOR}=4.63,95 \% \mathrm{CI}: 1.22-17.57, P=$ 0.024). In addition, males, participants with divorced or widowed and $\geq 3$ illegal drug use criminal record were also higher risk of unemployment $(P<0.001)$, but age and type of residence were not associated with subsequent employment status (Table 4).

\section{Collinearity diagnosis}

All the VIFs for models less than 10 (Tables 2, 3 and 4) and condition index less than 30 (Table 5) in this study, the results showed mild collinearity problem in our study models.

\section{Discussion}

In the present study, after controlling potential confounders (age, insurance premium at 2 years after index date, education level, marital status, illegal drug use criminal record, Charlson comorbidity score), the unemployment rate of illegal heroin users sentenced to prison was higher than that for those who received deferred prosecution, observation and rehabilitation and compulsory rehabilitation. These results indicate that, in the 2 years after release from the penal system, sentencing to prison made it more difficult for illegal heroin users to acquire a job compared to counterparts of the other three types. Employment is a critical component of the reentry process that positively assists illegal drug users to return to the community. The results support the viewpoint that a deferred prosecution policy can decrease the risk of unemployment and subsequently improve offenders' social control, economic conditions and ability to return to society. Continued implementation of a deferred prosecution and complete MMT treatment therefore benefits the offender and solve the problem of prison overcrowding.

Employment is a fundamental element of social control, providing a daily routine and expectations regarding performance and productivity. Whether illegal substance use disrupts that process and is associated with negative outcomes is therefore a critical social, economic, and criminal justice question, with implications for drug policy. Although the importance of the issue is widely recognized, the strength and direction of the relationship remains unclear. Early research often reported that drug use is unrelated to employment outcomes [36, 37] and, in some instances, that drug use is associated with a higher wages [38]. In contrast, research conducted post2000 reveals, more often than not, that drug use is associated with poor employment outcomes. Huang et al. 
Table 1 Demographic and other characteristics of study participants, Taiwan, $2011(n=9120)$

\begin{tabular}{|c|c|c|c|}
\hline \multirow[t]{2}{*}{ Characteristics } & \multicolumn{2}{|c|}{ Insurance premium at two years after index date ${ }^{a}$} & \multirow{2}{*}{$\begin{array}{l}P \\
\text { value }\end{array}$} \\
\hline & No job salary, $n=1848$ & With job salary, $n=7272$ & \\
\hline \multicolumn{4}{|l|}{ Gender } \\
\hline Male & $1524(20.20)$ & $6019(79.80)$ & \multirow[t]{2}{*}{0.759} \\
\hline Female & $324(20.55)$ & $1253(79.45)$ & \\
\hline \multicolumn{4}{|l|}{ Age (years) at index date } \\
\hline $18-24$ & $375(23.32)$ & $1233(76.68)$ & \multirow[t]{3}{*}{$<0.001$} \\
\hline $25-44$ & $1160(18.44)$ & $5131(81.56)$ & \\
\hline$\geq 45$ & $313(25.63)$ & $908(74.37)$ & \\
\hline \multicolumn{4}{|l|}{ Type of residence } \\
\hline Rural & $539(21.43)$ & $1976(78.57)$ & \multirow[t]{2}{*}{0.087} \\
\hline Urban & $1309(19.82)$ & $5296(80.18)$ & \\
\hline \multicolumn{4}{|c|}{ Insurance premium at two years after index date } \\
\hline With job salary & $1265(16.01)$ & $6635(83.99)$ & \multirow[t]{2}{*}{$<0.001$} \\
\hline Without job salary & $583(47.79)$ & $637(52.21)$ & \\
\hline \multicolumn{4}{|l|}{ Type of sentencing } \\
\hline Deferred prosecution & $393(16.33)$ & $2013(83.67)$ & \multirow[t]{4}{*}{$<0.001$} \\
\hline Observation and rehabilitation & $853(17.99)$ & $3888(82.01)$ & \\
\hline Compulsory rehabilitation & $6(40.00)$ & $9(60.00)$ & \\
\hline Sentenced to prison & $596(30.44)$ & $1362(69.56)$ & \\
\hline \multicolumn{4}{|l|}{ Education level } \\
\hline Elementary & $323(21.72)$ & $1164(78.28)$ & \multirow[t]{4}{*}{$<0.001$} \\
\hline Junior high school & $1107(21.47)$ & $4050(78.53)$ & \\
\hline Senior high school & $383(16.89)$ & $1885(83.11)$ & \\
\hline College & $35(16.83)$ & $173(83.17)$ & \\
\hline \multicolumn{4}{|l|}{ Marital status } \\
\hline Single & $1335(19.30)$ & $5581(80.70)$ & \multirow[t]{3}{*}{$<0.001$} \\
\hline Married & $348(21.97)$ & $1236(78.03)$ & \\
\hline Divorce or widowed & $165(26.61)$ & $455(73.39)$ & \\
\hline \multicolumn{4}{|l|}{ Drug use criminal record } \\
\hline 0 & $236(18.02)$ & $1074(81.98)$ & \multirow[t]{4}{*}{$<0.001$} \\
\hline 1 & $559(15.23)$ & $3111(84.77)$ & \\
\hline 2 & $342(19.94)$ & $1373(80.06)$ & \\
\hline$\geq 3$ & $711(29.32)$ & $1714(70.68)$ & \\
\hline \multicolumn{4}{|l|}{ Schizophrenia } \\
\hline Yes & $1822(20.31)$ & $7147(79.69)$ & \multirow[t]{2}{*}{0.321} \\
\hline No & $26(17.22)$ & $125(82.78)$ & \\
\hline \multicolumn{4}{|l|}{ Depression } \\
\hline Yes & $1675(20.20)$ & $6616(79.80)$ & \multirow[t]{2}{*}{0.687} \\
\hline No & $173(20.87)$ & $656(79.13)$ & \\
\hline Bipolar & & & \\
\hline Yes & $1819(20.22)$ & 7179 (79.78) & 0.381 \\
\hline No & $29(23.77)$ & $93(76.23)$ & \\
\hline Charlson comorbidity score & & & \\
\hline 0 & $915(21.43)$ & $3354(78.57)$ & $<0.001$ \\
\hline 1 & $446(18.82)$ & $1924(81.18)$ & \\
\hline 2 & $206(16.78)$ & $1022(83.22)$ & \\
\hline$\geq 3$ & $281(22.43)$ & $972(77.57)$ & \\
\hline
\end{tabular}

\footnotetext{
${ }^{a}$ The index date was the date of release from prison, treatment centers or deferred prosecution; job status monitoring lasted for two years after the index date
} 
Table 2 Logistic regression analysis of odds of unemployment at two years after release following heroin use sentence in Taiwan, $2011(n=9120)$

\begin{tabular}{|c|c|c|c|c|c|}
\hline \multirow[t]{2}{*}{ Variable } & \multicolumn{2}{|c|}{ Un-adjusted odds-ratio } & \multicolumn{2}{|c|}{ Adjusted odds-ratio $^{b}$} & \multirow[t]{2}{*}{ VIF $^{c}$} \\
\hline & $\begin{array}{l}\text { Estimate } \\
(95 \% \mathrm{Cl})\end{array}$ & $P$ value & $\begin{array}{l}\text { Estimate } \\
(95 \% \mathrm{Cl})\end{array}$ & $P$ value & \\
\hline \multicolumn{6}{|l|}{ Social demography $^{d}$} \\
\hline \multicolumn{6}{|l|}{ Gender } \\
\hline Male & 1.00 & & 1.00 & & \\
\hline Female & $1.02(0.89-1.17)$ & 0.758 & $0.75(0.64-0.87)$ & $<0.001$ & 1.1 \\
\hline \multicolumn{6}{|l|}{ Age (years) at index date ${ }^{a}$} \\
\hline $18-24$ & 1.00 & & 1.00 & & \\
\hline $25-44$ & $0.74(0.65-0.85)$ & $<0.001$ & $1.41(1.17-1.70)$ & $<0.001$ & 2.2 \\
\hline$\geq 45$ & $1.13(0.95-1.35)$ & 0.155 & $1.55(1.21-1.98)$ & $<0.001$ & 2.3 \\
\hline \multicolumn{6}{|l|}{ Type of residence } \\
\hline Rural & 1.00 & & 1.00 & & \\
\hline Urban & $0.91(0.81-1.01)$ & 0.087 & $0.91(0.80-1.03)$ & 0.131 & 1.0 \\
\hline \multicolumn{6}{|c|}{ Insurance premium at 2 years before index date } \\
\hline With job salary & 1.00 & & 1.00 & & \\
\hline Without job salary & $4.8(4.23-5.45)$ & $<0.001$ & $6.65(5.67-7.82)$ & $<0.001$ & 1.2 \\
\hline \multicolumn{6}{|l|}{ Education level } \\
\hline Elementary & 1.00 & & & & \\
\hline Junior high school & $0.99(0.85-1.14)$ & 0.844 & $1.06(0.91-1.25)$ & 0.448 & 2.0 \\
\hline Senior high school & $0.73(0.62-0.87)$ & $<0.001$ & $0.82(0.68-1.00)$ & 0.049 & 2.2 \\
\hline College & $0.73(0.49-1.09)$ & 0.122 & $0.82(0.54-1.25)$ & 0.358 & 1.1 \\
\hline \multicolumn{6}{|l|}{ Marital status } \\
\hline Single & 1.00 & & 1.00 & & \\
\hline Married & $1.18(1.03-1.35)$ & 0.019 & $1.06(0.9-1.24)$ & 0.500 & 1.2 \\
\hline Divorced or widowed & $1.52(1.25-1.84)$ & $<0.001$ & $1.42(1.13-1.78)$ & 0.003 & 1.2 \\
\hline \multicolumn{6}{|c|}{ Drug use sentences and criminal record ${ }^{d}$} \\
\hline \multicolumn{6}{|l|}{ Type of sentencing } \\
\hline Deferred prosecution & 1.00 & & 1.00 & & \\
\hline Observation and rehabilitation & $1.12(0.99-1.28)$ & 0.081 & $1.31(1.13-1.52)$ & $<0.001$ & 1.6 \\
\hline Compulsory rehabilitation & $2.24(1.94-2.59)$ & $<0.001$ & $1.87(1.59-2.20)$ & $<0.001$ & 1.5 \\
\hline Sentenced to prison & $3.41(1.21-9.65)$ & 0.021 & $2.30(1.97-2.65)$ & $<0.001$ & 1.0 \\
\hline \multicolumn{6}{|l|}{ Drug use criminal record } \\
\hline 0 & 1.00 & & 1.00 & & \\
\hline 1 & $0.86(0.69-1.07)$ & 0.121 & $0.88(0.73-1.06)$ & 0.171 & 2.3 \\
\hline 2 & $1.13(0.94-1.37)$ & 0.205 & $1.15(0.93-1.4)$ & 0.191 & 1.9 \\
\hline$\geq 3$ & $1.88(1.59-2.23)$ & $<0.001$ & $1.90(1.56-2.32)$ & $<0.001$ & 2.5 \\
\hline \multicolumn{6}{|l|}{ Mental diseases and comorbidity ${ }^{d}$} \\
\hline Schizophrenia (Yes vs No) & $0.80(0.51-1.24)$ & 0.322 & $0.86(0.54-1.39)$ & 0.542 & 1.1 \\
\hline Depression (Yes vs No) & $1.04(0.87-1.25)$ & 0.684 & $1.08(0.87-1.34)$ & 0.509 & 1.3 \\
\hline Bipolar (Yes vs No) & $1.21(0.79-1.87)$ & 0.381 & $1.33(0.82-2.16)$ & 0.242 & 1.1 \\
\hline \multicolumn{6}{|l|}{ Charlson comorbidity score } \\
\hline 0 & 1.00 & & 1.00 & & \\
\hline 1 & $0.94(0.85-1.07)$ & 0.115 & $0.90(0.79-1.04)$ & 0.153 & 1.2 \\
\hline 2 & $0.94(0.82-1.08)$ & 0.111 & $0.80(0.66-0.96)$ & 0.017 & 1.2 \\
\hline$\geq 3$ & $1.06(0.91-1.24)$ & 0.460 & $0.99(0.82-1.18)$ & 0.888 & 1.3 \\
\hline
\end{tabular}

The index date was the date of release from prison, treatment centers or deferred prosecution

${ }^{b}$ Global model fit Wald test $x^{2}=807.6, \mathrm{DF}=22, P<0.001 ; \mathrm{R}$-square $=0.154$

c VIF Variance inflation factor

${ }^{d}$ For controlling potential confounders, social demography were collected from National Population Register Database, including gender, age, types of residence, insurance premium, education level and marital status [22, 30,31]. Illicit drug use criminal record collected from National Police Agency in Taiwan [32, 33]. Mental diseases including schizophrenia, depressive disorders, bipolar disorders and general health condition (Charlson comorbidity score) were collected from NHIRD [31, 34, 35] 
Table 3 Logistic regression analysis of odds of unemployment at two years after release following heroin use sentence in Taiwan for those unemployed before sentencing, $2011(n=1220)$

\begin{tabular}{|c|c|c|c|c|c|}
\hline \multirow[t]{2}{*}{ Variable } & \multicolumn{2}{|c|}{ Un-adjusted odds-ratio } & \multicolumn{2}{|c|}{ Adjusted odds-ratio $^{b}$} & \multirow[t]{2}{*}{$\mathrm{VIF}^{\mathrm{c}}$} \\
\hline & $\begin{array}{l}\text { Estimate } \\
(95 \% \mathrm{Cl})\end{array}$ & $P$ value & $\begin{array}{l}\text { Estimate } \\
(95 \% \mathrm{Cl})\end{array}$ & $P$ value & \\
\hline \multicolumn{6}{|l|}{ Social demography $^{\text {d }}$} \\
\hline \multicolumn{6}{|l|}{ Gender } \\
\hline Male & 1.00 & & 1.00 & & \\
\hline Female & $0.94(0.74-1.19)$ & 0.610 & $0.97(0.74-1.26)$ & 0.815 & 1.1 \\
\hline \multicolumn{6}{|l|}{ Age (years) at index date ${ }^{a}$} \\
\hline $18-24$ & 1.00 & & 1.00 & & \\
\hline $25-44$ & $1.79(1.40-2.3)$ & $<0.001$ & $1.98(1.40-2.80)$ & $<0.001$ & 1.9 \\
\hline$\geq 45$ & $4.34(2.9-6.49)$ & $<0.001$ & $5.10(2.79-9.35)$ & $<0.001$ & 2.3 \\
\hline \multicolumn{6}{|l|}{ Type of residence } \\
\hline Rural & 1.00 & & 1.00 & & \\
\hline Urban & $0.88(0.68-1.15)$ & 0.345 & $0.87(0.65-1.15)$ & 0.331 & 1.0 \\
\hline \multicolumn{6}{|l|}{ Education level } \\
\hline Elementary & 1.00 & & 1.00 & & \\
\hline Junior high school & $0.87(0.66-1.14)$ & 0.311 & $0.87(0.65-1.16)$ & 0.348 & 1.4 \\
\hline Senior high school & $1.37(0.92-2.05)$ & 0.118 & $0.91(0.57-1.46)$ & 0.706 & 1.7 \\
\hline College & $1.03(0.40-2.66)$ & 0.956 & $0.59(0.21-1.64)$ & 0.312 & 1.1 \\
\hline \multicolumn{6}{|l|}{ Marital status } \\
\hline Single & 1.00 & & 1.00 & & \\
\hline Married & $1.89(1.38-2.59)$ & $<0.001$ & $0.87(0.57-1.33)$ & 0.529 & 1.7 \\
\hline Divorced or widowed & $2.43(1.38-4.29)$ & 0.002 & $0.95(0.47-1.90)$ & 0.888 & 1.5 \\
\hline \multicolumn{6}{|c|}{ Drug use Sentences and criminal record ${ }^{d}$} \\
\hline \multicolumn{6}{|l|}{ Type of sentencing } \\
\hline Deferred prosecution & 1.00 & & 1.00 & & \\
\hline Observation and rehabilitation & $0.89(0.67-1.18)$ & 0.414 & $1.19(0.86-1.63)$ & 0.287 & 1.6 \\
\hline Compulsory rehabilitation & $0.71(0.12-4.33)$ & 0.711 & $0.90(0.13-6.15)$ & 0.914 & 1.6 \\
\hline Sentenced to prison & $1.34(0.93-1.94)$ & 0.118 & $1.04(0.68-1.60)$ & 0.849 & 1.0 \\
\hline \multicolumn{6}{|l|}{ Drug use criminal record } \\
\hline 0 & 1.00 & & 1.00 & & \\
\hline 1 & $0.90(0.65-1.25)$ & 0.534 & $0.89(0.63-1.26)$ & 0.513 & 2.0 \\
\hline 2 & $1.11(0.76-1.61)$ & 0.595 & $1.00(0.67-1.49)$ & 0.984 & 1.8 \\
\hline$\geq 3$ & $1.05(0.73-1.53)$ & 0.787 & $0.86(0.57-1.29)$ & 0.464 & 2.0 \\
\hline \multicolumn{6}{|l|}{ Mental diseases and comorbidity ${ }^{d}$} \\
\hline Schizophrenia (Yes VS No) & $3.22(0.33-6.00)$ & 0.312 & $1.17(0.11-2.09)$ & 0.894 & 1.1 \\
\hline Depression (Yes VS No) & $2.41(1.51-3.84)$ & $<0.001$ & $2.44(1.42-4.19)$ & 0.001 & 1.3 \\
\hline Bipolar (Yes VS No) & $1.94(0.65-5.84)$ & 0.236 & $0.79(0.22-2.80)$ & 0.720 & 1.2 \\
\hline \multicolumn{6}{|l|}{ Charlson comorbidity score } \\
\hline 0 & 1.00 & & 1.00 & & \\
\hline 1 & $1.15(0.87-1.52)$ & 0.336 & $1.11(0.83-1.49)$ & 0.480 & 1.1 \\
\hline 2 & $1.03(0.70-1.52)$ & 0.871 & $0.90(0.60-1.36)$ & 0.626 & 1.1 \\
\hline$\geq 3$ & $1.87(1.26-2.79)$ & 0.002 & $1.14(0.71-1.84)$ & 0.590 & 1.4 \\
\hline
\end{tabular}

The index date was the date of release from prison, treatment centers or deferred prosecution

b Global model fit Wald test $x^{2}=72.28, D F=21, P<0.001 ; R$-square $=0.093$

${ }^{c}$ VIF Variance inflation factor

${ }^{d}$ For controlling potential confounders, social demography were collected from National Population Register Database, including gender, age, types of residence, insurance premium, education level and marital status [22, 30, 31]. Illicit drug use criminal record collected from National Police Agency in Taiwan [32, 33]. Mental diseases including schizophrenia, depressive disorders, bipolar disorders and general health condition (Charlson comorbidity score) were collected from NHIRD $[31,34,35]$ 
Table 4 Logistic regression analysis of risk of unemployment at two years after release following heroin use sentence in Taiwan among those employed before sentence, $2011(n=7900)$

\begin{tabular}{|c|c|c|c|c|c|}
\hline \multirow[t]{2}{*}{ Variable } & \multicolumn{2}{|c|}{ Un-adjusted odds-ratio } & \multicolumn{2}{|c|}{ Adjusted odds-ratio $^{\text {b }}$} & \multirow[t]{2}{*}{${ }_{c}^{\text {VIF }}$} \\
\hline & $\begin{array}{l}\text { Estimate } \\
(95 \% \mathrm{Cl})\end{array}$ & $P$ value & $\begin{array}{l}\text { Estimate } \\
(95 \% \mathrm{Cl})\end{array}$ & $P$ value & \\
\hline \multicolumn{6}{|l|}{ Social demography $^{\text {d }}$} \\
\hline \multicolumn{6}{|l|}{ Gender } \\
\hline Male & 1.00 & & 1.00 & & \\
\hline Female & $0.61(0.5-0.74)$ & $<0.001$ & $0.63(0.51-0.77)$ & $<0.001$ & 1.1 \\
\hline \multicolumn{6}{|l|}{ Age (year) at index date ${ }^{a}$} \\
\hline $18-24$ & 1.00 & & 1.00 & & \\
\hline $25-44$ & $1.36(1.1-1.67)$ & 0.004 & $1.11(0.88-1.40)$ & 0.364 & 2.2 \\
\hline$\geq 45$ & $1.7(1.33-2.18)$ & $<0.001$ & $1.05(0.78-1.41)$ & 0.752 & 2.5 \\
\hline \multicolumn{6}{|l|}{ Type of residence } \\
\hline Rural & 1.00 & & 1.00 & & \\
\hline Urban & $0.85(0.75-0.97)$ & 0.018 & $0.91(0.77-1.12)$ & 0.208 & 1.0 \\
\hline \multicolumn{6}{|l|}{ Education level } \\
\hline Elementary & 1.00 & & 1.00 & & \\
\hline Junior high school & $1.29(1.07-1.56)$ & 0.007 & $1.22(1-1.48)$ & 0.051 & 2.2 \\
\hline Senior high school & $0.98(0.80-1.22)$ & 0.888 & $0.90(0.71-1.12)$ & 0.339 & 2.3 \\
\hline College & $0.95(0.60-1.51)$ & 0.825 & $0.94(0.58-1.51)$ & 0.791 & 1.2 \\
\hline \multicolumn{6}{|l|}{ Marital status } \\
\hline Single & 1.00 & & 1.00 & & \\
\hline Married & $1.07(0.90-1.26)$ & 0.452 & $1.06(0.89-1.27)$ & 0.517 & 1.2 \\
\hline Divorced or widowed & $1.60(1.29-1.98)$ & $<0.001$ & $1.51(1.19-1.94)$ & 0.001 & 1.2 \\
\hline \multicolumn{6}{|c|}{ Drug use Sentences and criminal record ${ }^{d}$} \\
\hline \multicolumn{6}{|l|}{ Type of sentencing } \\
\hline Deferred prosecution & 1.00 & & 1.00 & & \\
\hline Observation and rehabilitation & $1.02(0.87-1.19)$ & 0.841 & $1.38(1.16-1.65)$ & $<0.001$ & 1.5 \\
\hline Compulsory rehabilitation & $2.61(2.22-3.08)$ & $<0.001$ & $2.07(1.73-2.47)$ & $<0.001$ & 1.5 \\
\hline Sentenced to prison & $4.62(1.29-16.47)$ & 0.018 & $4.63(1.22-17.57)$ & 0.024 & 1.0 \\
\hline \multicolumn{6}{|l|}{ Drug use criminal record } \\
\hline 0 & 1.00 & & 1.00 & & \\
\hline 1 & $0.87(0.70-1.08)$ & 0.211 & $0.87(0.70-1.09)$ & 0.237 & 2.3 \\
\hline 2 & $1.25(0.98-1.59)$ & 0.070 & $1.19(0.93-1.52)$ & 0.161 & 1.9 \\
\hline$\geq 3$ & $2.67(2.16-3.03)$ & $<0.001$ & $2.30(1.82-2.90)$ & $<0.001$ & 2.6 \\
\hline \multicolumn{6}{|l|}{ Mental diseases and comorbidity ${ }^{d}$} \\
\hline Schizophrenia (Yes VS No) & $0.95(0.59-1.51)$ & 0.816 & $0.92(0.56-1.52)$ & 0.750 & 1.1 \\
\hline Depression (Yes VS No) & $0.91(0.73-1.14)$ & 0.413 & $0.88(0.68-1.13)$ & 0.313 & 1.3 \\
\hline Bipolar (Yes VS No) & $1.15(0.69-1.91)$ & 0.605 & $1.38(0.79-2.41)$ & 0.254 & 1.1 \\
\hline \multicolumn{6}{|l|}{ Charlson comorbidity score } \\
\hline 0 & 1.00 & & 1.00 & & \\
\hline 1 & $0.81(0.69-0.95)$ & 0.008 & $0.84(0.71-0.98)$ & 0.031 & 1.2 \\
\hline 2 & $0.73(0.59-0.89)$ & 0.002 & $0.77(0.62-0.94)$ & 0.013 & 1.2 \\
\hline$\geq 3$ & $1.06(0.88-1.27)$ & 0.529 & $0.94(0.77-1.15)$ & 0.569 & 1.3 \\
\hline
\end{tabular}

${ }^{\mathrm{a}}$ The index date was the date of release from prison, treatment centers or deferred prosecution

b Global model fit Wald test $x^{2}=368.5, D F=21, P<0.001 ; R$-square $=0.085$

c VIF Variance inflation factor

${ }^{d}$ For controlling potential confounders, social demography were collected from National Population Register Database, including gender, age, types of residence, insurance premium, education level and marital status [22, 30, 31]. Illicit drug use criminal record collected from National Police Agency in Taiwan [32, 33]. Mental diseases including schizophrenia, depressive disorders, bipolar disorders and general health condition (Charlson comorbidity score) were collected from NHIRD $[31,34,35]$ 
Table 5 Condition index to check collinearity for the models of Tables 2, 3 and 4 in this study

\begin{tabular}{|c|c|c|c|}
\hline \multirow[t]{2}{*}{ Number } & \multicolumn{3}{|l|}{ Condition Index } \\
\hline & Model of Table 2 & Model of Table 3 & Model of Table 4 \\
\hline 1 & 1.0 & 1.0 & 1.0 \\
\hline 2 & 2.0 & 1.9 & 2.0 \\
\hline 3 & 2.3 & 2.1 & 2.3 \\
\hline 4 & 2.5 & 2.2 & 2.5 \\
\hline 5 & 2.6 & 2.4 & 2.7 \\
\hline 6 & 2.7 & 2.5 & 2.7 \\
\hline 7 & 2.7 & 2.6 & 2.7 \\
\hline 8 & 2.7 & 2.6 & 2.7 \\
\hline 9 & 2.8 & 2.6 & 2.8 \\
\hline 10 & 2.8 & 2.7 & 2.8 \\
\hline 11 & 2.8 & 2.8 & 2.8 \\
\hline 12 & 2.9 & 2.9 & 2.9 \\
\hline 13 & 3.0 & 3.0 & 3.0 \\
\hline 14 & 3.0 & 3.2 & 3.1 \\
\hline 15 & 3.1 & 3.4 & 3.7 \\
\hline 16 & 3.7 & 3.6 & 3.8 \\
\hline 17 & 3.8 & 3.8 & 3.9 \\
\hline 18 & 3.9 & 4.1 & 4.4 \\
\hline 19 & 4.4 & 5.4 & 5.3 \\
\hline 20 & 5.4 & 7.3 & 8.9 \\
\hline 21 & 8.4 & 9.3 & 10.5 \\
\hline 22 & 10.3 & 19.4 & 21.8 \\
\hline 23 & 21.4 & - & - \\
\hline
\end{tabular}

[24] find that use of heroin as well as higher frequency use is predictive of membership in a low-employment trajectory group through middle adulthood. The present study extended these findings and further showed that higher illegal drug use criminal record ( $>3$ times) was associated with a risk of unemployment after controlling potential confounders, especially those who had a job before sentencing.

As well, DeSimone [39], adopting a sophisticated econometric model that addresses simultaneity, reports a negative relationship between illegal drug use, employment, and labor force participation [40]. Problematic substance use increases the likelihood of unemployment and decreases the chance of finding and holding down a job. Unemployment is also a significant risk factor for substance use and the subsequent development of substance use disorders. Unemployment and substance use have a bidirectional correlation [41]; in other words, improving the employment rate in the illegal drug use population contribute to reduce the recurrence of drug use. Moreover, employment is highly associated with treatment retention [42, 43]. Thus, the deferred sentencing model can decrease the unemployment rate and assist former drug users continue to receive treatment. Employment helps users maintain their treatment and recover better.

A previous study revealed that former employment and work experiences are protective factors that increase the likelihood of employment after release [44]. Similarly, our data showed that the unemployment rate for illegal heroin users without a job before the index date was higher than that for those with a job before the index data. Interestingly, those sentenced to prison had a higher risk of unemployment than those who received deferred prosecution, observation and rehabilitation and compulsory rehabilitation, even if they were employed before being arrested (Tables 3 and 4). This result suggests that illegal heroin use treatment for those with prior employment might be superior in the criminal justice settings to sentencing only, but not for those previously were unemployed. Reducing the number of people incarcerated for illegal drug use can net huge savings in economic and social costs [45]. The finding support that the policy of prioritizing treatment for illegal heroin user over imprisonment deserves continued promotion.

Previous studies have indicated that gender and age are associated with employment status in illegal heroin user [24, 46]. At ages 23-25, the low-employment trajectory group was employed in approximately 21 of 52 workweeks, followed by a rapid decline to 10 or fewer by age 40 [24]. Similarly, we also found that being male and being older increased the risk of unemployment in illegal heroin users at 2 years after release. Furthermore, we found that the association of gender and employment status remained significant for those previously were employed, while the association of age and employment status was still significant for those previously were unemployed. This difference suggests that previously unemployed older heroin users and previously employed male users were more likely to be jobless after arrest, increasing their likelihood of unemployment after their release from the system.

A illegal drug use crime refers to a detrimental behavior or an illness, society has a obligation to expect that an effective public policy or approach to the "illegal drug use problem" that will reduce drug-related crime, unemployment, family dysfunction and disproportionate use of heath care [47]. However, although half of prisoners need treatment, approximately $14.8-17.4 \%$ reported having received drug treatment since admission in a survey of US State and Federal prisoners, according to the Bureau of Justice Statistics (BJS) [48]. Another study was conducted to compare rates of employment before, during, and after employment at the therapeutic workplace [49]. The researchers found that unemployed chronic drug users will attend work at higher rates at 
the therapeutic workplace than in the community when paid modest wages. Thus, after receiving treatment, a successful mechanism that can transfer the illegal drug user to the therapeutic workplace contribute to improve their employment rate. Providing vocational training as part of drug abstinence-oriented treatment has the potential to improve employment-seeking behaviors, increase the chances of securing employment, improve the employment rate and lead to higher paying jobs [48]. Consequently, a high quality treatment program is necessary and should produce positive results.

To our knowledge, this is the first national populationbased study to investigate the association of type of sentencing and the subsequent employment status among illegal heroin users in Taiwan. The major strength of this study is its population-based study design. Because this study was conducted using the population-based database, the findings can be applied to the general population. However, this study has several limitations. Several crucial confounding factors could not be included in this study, including severity of the substance use disorder, family income and social support system since our study database did not contain this information. Second, we only track the employment status of illegal heroin users at 2 years after release, if more time points of employment status can be included in the analyses, generalizability of study findings will be warrant. Third, for drug users, three factors associated with unemployed were lower education, the personal willing of discouraged workers, and social discrimination. As Webster et al. (2007) addressed that all of the employers surveyed were willing to hire someone having no criminal record before hiring a released prisoner [33]. However, there is no relevant survey variable in the database, the influences of these three factors did not include in this analyses. Interpretation of the study results should be with caution.

\section{Conclusion}

Basically, illegal heroin users are legally regarded as a patient before being regarded as a criminal, so giving priority to quit addition rather than imprisonment. This study supports this viewpoint that staying in the community to receive methadone maintenance treatment (MMT) may increase the odds ratio of illegal heroin users who keep their jobs. But for illegal heroin users who were unemployed before their arrest would not be significantly different because of four different sentencing types. Illegal heroin users sent to prison may be at greater risk for subsequent unemployment than those receiving deferred prosecution, observation and rehabilitation, and compulsory rehabilitation, especially among those who had a job before sentencing. The implication is the stronger freedom of punishment, the higher risk of unemployment outcomes. In addition, males, older offenders, those without a job before sentencing, divorced or widowed, those have $\geq 3$ illegal drug use criminal records are also at higher risk of unemployment at 2 years after release. Effective public policy should strive to increase the employability of this population, in order to reduce both illegal drug use and prison sentencing in society as a whole. However, such a conclusion of the causal relationship between the deferred prosecution policy and the risk of unemployment should be proved by the social experiments or other methods for further research.

\section{Abbreviations \\ BJS: Bureau of Justice Statistics; HIV: Human immunodeficiency virus; IRB: Institutional Review Board; MMT: Methadone maintenance treatment; NHI: National Health Insurance; NHIRD: NHI Research Database}

\section{Acknowledgments}

This research was supported by the Taiwan Food and Drug Administration (105TFDA-N-005). Dr. Charles Tzu Chi Lee had full access to all the data in the study and takes responsibility for the data integrity and the accuracy of the data analysis.

\section{Authors' contributions}

Charles Tzu Chi Lee, Chiu-Mieh Huang and Jong-Long Guo contributed to the study concept and design, and analysis and interpretation of data; Charles Tzu Chi Lee, Chiu-Mieh Huang, Shih-Wen Wang, Hsiao-Pei Hsu and Jong-Long Guo drafted of the manuscript; Li-Chun Chang, Shih-Wen Wang, Jung-Yu Liao and Hsiao-Pei Hsu contributed to data acquisition; Charles Tzu Chi Lee, Chiu-Mieh Huang, Li-Chun Chang, and Jong-Long Guo performed the critical revision of the manuscript; all authors agreed with the final manuscript.

\section{Funding}

This research was supported by the Taiwan Food and Drug Administration (105TFDA-N-005).

\section{Availability of data and materials}

The data used to support the findings of this study are included within the article.

\section{Ethics approval and consent to participate}

The Institutional Review Board (IRB) of National Taiwan Normal University approved this study (No. 201606HM002). Written consent from the study participants was waived because the data were collected from a populationbased database of de-identified secondary data.

\section{Consent for publication}

Not applicable.

\section{Competing interests}

The authors declare that they have no competing interests.

\section{Author details}

${ }^{1}$ Department of Health Promotion and Health Education, National Taiwan Normal University, 162, Sec. 1, He-ping East Road, Taipei, Taiwan 10610. ${ }^{2}$ Institute of Clinical Nursing, School of Nursing, National Yang-Ming University, 155, Sec. 2, Li-Nong Street, Taipei, Taiwan 11221. 3Department of Nursing, Chang Gung University of Science and Technology, 261, Wen-Hua 1st Rd, Tao-Yuan, Taiwan 33303. ${ }^{4}$ Institute of Population Health Sciences, National Health Research Institutes (NHRI), 35 Keyan Road, Zhunan, Miaoli County 35053, Taiwan. 


\section{Accepted: 2 October 2020}

Published online: 12 October 2020

\section{References}

1. Khalsa JH, Genser S, Francis H, Martin B. Clinical consequences of marijuana. J Clin Pharmacol. 2002;42(S1):7S-10S.

2. Humensky JL, Jordan N, Stroupe KT, Hynes D. Employment status of veterans receiving substance abuse treatment from the U.S. Department of Veterans Affairs. Psychiatr Serv. 2013;64(2):177-80.

3. Chandler RK, Fletcher BW, Volkow ND. Treating drug abuse and addiction in the criminal justice system: improving public health and safety. JAMA. 2009; 301(2):183-90

4. Dunigan R, Acevedo A, Campbell K, Garnick DW, Horgan CM, Huber A, et al. Engagement in outpatient substance abuse treatment and employment outcomes. J Behav Health Serv Res. 2014:41(1):20-36.

5. United Nations Office on Drugs and Crime. World drug report 2020. Vienna: United Nations publication; 2020.

6. Yu WJ, Tsay WI, Li JH. Current status of substance abuse and HIV in Taiwan. J Food Drug Anal. 2013;21(4):S27-32.

7. United Nations Office on Drugs and Crime. World drug report 2012. Vienna: United Nations publication; 2012

8. Hsu J, Lin JJ, Tsay WI. Analysis of drug abuse data reported by medical institutions in Taiwan from 2002 to 2011. J Food Drug Anal. 2014;22:169-77.

9. Rice DP. Economic costs of substance abuse, 1995. Proc Assoc Am Physicians. 1999;111(2):119-25.

10. Mark TL, Woody GE, Juday T, Kleber HD. The economic costs of heroin addiction in the United States. Drug Alcohol Depend. 2001;61(2):195-206.

11. Healey A, Knapp M, Astin J, Gossop M, Marsden J, Stewart D, et al. Economic burden of drug dependency. Social costs incurred by drug users at intake to the National Treatment Outcome Research Study. Br J Psychiatry. 1998;173:160-5.

12. Booth RE, Crowley TJ, Zhang Y. Substance abuse treatment entry, retention and effectiveness: out-of-treatment opiate injection drug users. Drug Alcohol Depend. 1996;42(1):11-20.

13. McLellan AT, Arndt IO, Metzger DS, Woody GE, O'Brien CP. The effects of psychosocial services in substance abuse treatment. JAMA. 1993;269(15): 1953-9.

14. Center for Substance Abuse Treatment. Substance Abuse Treatment for Adults in the Criminal Justice System. In: Substance Abuse and Mental Health Services Administration (US). Rockville: SAMHSA/CSAT Treatment Improvement Protocols (TIP) Series N Report No: (SMA) 05-4056; 2005.

15. Ministry of Justice. Narcotics Hazard Prevention Act. 2020. https://law.moj. gov.tw/ENG/LawClass/LawAll.aspx?pcode=C0000008. Accessed 1 Sep 2020

16. Lee MC. The study on exploring possible coping for the Prisoner's structure in correctional institutions. J Corrections. 2017;6(1):5-39.

17. Leufstadius C, Eklund M, Erlandsson LK. Meaningfulness in work experiences among employed individuals with persistent mental illness. Work. 2009:34(1):21-32

18. Hall AC, Kramer J. Social capital through workplace connections: opportunities for workers with intellectual disabilities. J Soc Work Disabil Rehabil. 2009;8(3-4):146-70.

19. Zaninotto P, Falaschetti E, Sacker A. Age trajectories of quality of life among older adults: results from the English longitudinal study of ageing. Qual Life Res. 2009:18(10):1301-9.

20. Virtanen P, Vahtera J, Broms U, Sillanmaki L, Kivimaki M, Koskenvuo M. Employment trajectory as determinant of change in health-related lifestyle: the prospective HeSSup study. Eur J Pub Health. 2008;18(5):504-8.

21. Zabkiewicz D. The mental health benefits of work: do they apply to poor single mothers? Soc Psychiatry Psychiatr Epidemiol. 2010;45(1):77-87.

22. Edwards AC, Ohlsson H, Sundquist J, Sundquist K, Kendler KS. Socioeconomic sequelae of drug abuse in a Swedish national cohort. Drug Alcohol Depend. 2020;212:107990.

23. Baldwin ML, Marcus SC, De Simone J. Job loss discrimination and former substance use disorders. Drug Alcohol Depend. 2010;110(1-2):1-7.

24. Huang DY, Evans E, Hara M, Weiss RE, Hser YI. Employment trajectories: exploring gender differences and impacts of drug use. J Vocat Behav. 2011; 79(1):277-89.

25. Naccarato T, Brophy M, Courtney ME. Employment outcomes of foste youth: the results from the Midwest evaluation of the adult functioning of Foster youth. Child Youth Serv Rev. 2010;32(4):551-9.
26. Kmec JA, Furstenberg FF Jr. Racial and gender differences in the transition to adulthood: a longitudinal study of Philadelphia youth. Adv Life Course Res. 2002;7:435-70.

27. Percheski C, Wildeman C. Becoming a dad: employment trajectories of married, cohabiting, and nonresident fathers. Soc Sci Q. 2008;89(2):482-501.

28. Charles KK, Decicca P. Local labor market fluctuations and health: is there a connection and for whom? J Health Econ. 2008;27(6):1532-50.

29. García-Gómez P, Jones AM, Rice N. Health effects on labour market exits and entries. Labour Econ. 2010;17(1):62-76.

30. Yu S-K, Chen K-T. Epidemiology of drug abuse from psychiatry hospitals in Taiwan. (January 1995-may 1996). J Clin Epidemiol. 1997:50:S34.

31. Zuelke AE, Luck T, Schroeter ML, Witte AV, Hinz A, Engel C, et al. The association between unemployment and depression-results from the population-based LIFE-adult-study. J Affect Disord. 2018;235:399-406.

32. Puigdollers E, Domingo-Salvany A, Brugal MT, Torrens M, Alvaros J, Castillo $C$, et al. Characteristics of heroin addicts entering methadone maintenance treatment: quality of life and gender. Subst Use Misuse. 2004;39(9):1353-68.

33. Webster M. Willingness of employers in the Denver metropolitan area to hire released prisoners. 2007. https://search.proquest.com/openview/6 88dd96c6c4fa1508e192e4063b84618/1?pq-origsite=gscholar\&cbl=1875 0\&diss=y. Accessed 1 Sep 2020

34. Frasquilho D, de Matos MG, Santos T, Gaspar T. Caldas de Almeida JM. Unemployment as a source of mental distress to individuals and their family: unemployed parents' perceptions during the economic recession. Int J Soc Psychiatry. 2016;62(5):477-86.

35. Büttner $\mathrm{A}$. The neuropathology of drug abuse. Curr Opin Behav Sci. 2017 ; 13(2):8-12.

36. French MT, Zarkin GA, Dunlap LJ. Illicit drug use, absenteeism, and earnings at six U.S. worksites. Contemp Econ Policy. 1998;16(3):334-46.

37. Zarkin GA, Mroz TA, Bray JW, French MT. The relationship between drug use and labor supply for young men. Labour Econ. 1998;5(4):385-409.

38. Register CA, Williams DR. Labor market effects of marijuana and cocaine use among young men. Ind Labor Relat Rev. 1992:45(3):435-48.

39. DeSimone J. Illegal drug use and employment. J Labor Econ. 2002;20(4): 952-77.

40. Arria AM, Garnier-Dykstra LM, Cook ET, Caldeira KM, Vincent KB, Baron RA, et al. Drug use patterns in young adulthood and post-college employment. Drug Alcohol Depend. 2013;127(1-3):23-30

41. Henkel D. Unemployment and substance use: a review of the literature (1990-2010). Curr Drug Abuse Rev. 2011;4(1):4-27.

42. Marcovitz DE, McHugh RK, Volpe J, Votaw V, Connery HS. Predictors of early dropout in outpatient buprenorphine/naloxone treatment. Am J Addict. 2016;25(6):472-7.

43. Weinstein ZM, Kim HW, Cheng DM, Quinn E, Hui D, Labelle CT, et al. Longterm retention in office based opioid treatment with buprenorphine. J Subst Abus Treat. 2017;74:65-70.

44. Visher CA, Debus-Sherrill SA, Yahner J. Employment after prison: a longitudinal study of former prisoners. Justice Q. 2011;28(5):698-718.

45. Zarkin GA, Dunlap $L$, Wedehase B, Cowell AJ. The effect of alternative staff time data collection methods on drug treatment service cost estimates. Eval Program Plann. 2008;31(4):427-35.

46. Richardson L, Wood E, Li K, Kerr T. Factors associated with employment among a cohort of injection drug users. Drug Alcohol Rev. 2010;29(3):293-300.

47. Hair JF, Sarstedt M, Ringle CM, Mena JA. An assessment of the use of partial least squares structural equation modeling in marketing research. J Acad Mark Sci. 2012;40(3):414-33.

48. Mumola CJ, Karberg JC. Drug use and dependence, state and federal prisoners, 2004. 2006. https://www.bjs.gov/content/pub/pdf/dudsfp04.pdf. Accessed 1 Sep 2020.

49. Sigurdsson SO, DeFulio A, Long L, Silverman K. Propensity to work among chronically unemployed adult drug users. Subst Use Misuse. 2011:46(5):599-607.

\section{Publisher's Note}

Springer Nature remains neutral with regard to jurisdictional claims in published maps and institutional affiliations. 